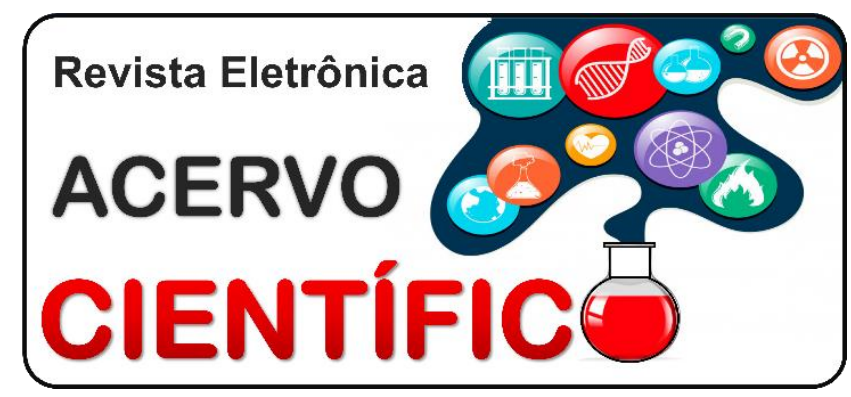

REVISÃO BIBLIOGRÁFICA

\title{
Impactos da obesidade em condições reumatológicas e na saúde discal
}

\author{
Impacts of obesity on rheumatotological conditions and discal health
}

Impactos de la obesidad en afecciones reumatológicas y en la salud discal

Isabella Ferraz Ferreira ${ }^{1 *}$, Ricardo André dos Santos $V_{a z}^{2}$, Ana Vitória Rodrigues de Sá ${ }^{3}$, André Teles da Silva4, Emílio Roberto Valiatti Zanotti ${ }^{4}$, Loan Langowski dos Santos ${ }^{4}$, Luis Eduardo Gonçalves Schmidt ${ }^{4}$, Marcela Moulin Magalhães ${ }^{5}$, Rebeca Magalhães Barros ${ }^{6}$, Vinícius Sippel Cruz ${ }^{7}$.

Resumo: Este artigo é uma revisão da literatura, que visa retratar consequências da obesidade, uma doença metabólica crônica, em condições reumatológicas e discais, utilizando resultados obtidos em artigos publicados nas bases de dados LILACS, MEDLINE, PUBMED e SCIELO. A obesidade é considerada um grande desafio à saúde pública, existente tanto em países subdesenvolvidos quanto desenvolvidos. Tal panorama se justifica pelos maus hábitos alimentares e sedentarismo, observados em grande parte da população global. É uma doença multissistêmica, na qual se encontram implicações inflamatórias e imunomoduladoras que prejudicam o equilíbrio entre os perfis pró e anti-inflamatório da resposta imunológica. Essas alterações exercem influência não somente na gênese das complicações, mas também na falência do tratamento e redução nos índices de remissão. Há elevação dos níveis de marcadores inflamatórios e alteração da progressão de outras comorbidades, de forma que a obesidade se apresenta como fator agravante de doenças reumatológicas e musculoesqueléticas. Adequação alimentar e prática de exercícios são indicados, pois levam à perda de peso, com consequente redução de sobrecarga mecânica, dos biomarcadores inflamatórios e do estresse oxidativo.

Palavras-chave: Obesidade, Inflamação, Doenças Reumáticas.

Abstract: This article is a literature review, that aims to evidence consequences of obesity, which is a chronic metabolic disease, on rheumatological and discal conditions, using results obtained in articles published on LILACS, MEDLINE, PUBMED and SCIELO databases. Obesity is considered a major public health challenge, existing in both developed and underdeveloped countries. This panorama is justified by the poor eating habits and sedentary lifestyle, observed in the majority of global population. It is a mutisystemic disease, in which are observed inflammatory and immunomodulatory implications, impairing the balance between the pro and anti-inflammatory immune response profiles. These alterations influence not only the genesis of complications, but also treatment failure and decreased remission rates. There is an increase of inflammatory markers and alterations in other comorbidities progression, so that obesity presents itself as an aggravating factor for rheumatic and musculoskeletal diseases. Dietary adequacy and physical exercise are indicated, leading to weight loss, with consequent reduction of mechanical overloading, inflammatory biomarkers and oxidative stress.

Keywords: Obesity, Inflammation, Rheumatic Diseases.

\footnotetext{
${ }^{1}$ Universidade de Vassouras (UV), Vassouras - RJ. *E-mail: ferrazisabella@hotmail.com

2 Universidade do Estado do Rio de Janeiro (UERJ), Rio de Janeiro - RJ.

${ }^{3}$ Universidade de Uberaba (UNIUBE), Uberaba - MG.

${ }^{4}$ Universidade Estácio de Sá (UNESA), Rio de Janeiro - RJ.

${ }^{5}$ Faculdade de Medicina de Petrópolis (UNIFASE), Petrópolis - RJ.

${ }^{6}$ Universidade Fundação Oswaldo Aranha (UniFoa), Volta Redonda - RJ.

${ }^{7}$ Faculdade Evangélica Mackenzie do Paraná (FEMPAR), Curitiba - PR.
} 
Resumen: Este artículo es una revisión de la literatura, que tiene como objetivo retratar las consecuencias de la obesidade, una enfermedad metabólica crónica, en condiciones reumatológicas y discales, utilizando los resultados obtenidos en los artículos publicados en las bases de datos LILACS, MEDLINE, PUBMED y SCIELO. La obesidad es considerada un importante desafío de salud pública, existente tanto en países subdesarrollados como desarrollados. Este panorama se justifica por los malos hábitos alimentarios y la inactividad física, que se observa en gran parte de la población mundial. Es una enfermedad multisistémica, en la cual existen implicaciones inflamatorias e inmunomoduladores que alteran el equilibrio entre los perfiles pro y antiinflamatorios de la respuesta inmune. Estos cambios influyen no solo en la génesis de las complicaciones, sino también en el fracaso del tratamiento y la reducción de las tasas de remisión. Existe un aumento de los niveles de marcadores inflamatorios y cambios en la progresión de otras comorbilidades, por lo que la obesidad es un agravante de las enfermedades reumatológicas y musculoesqueléticas. Adecuación de la alimentación y el ejercicio están indicados, conducen a la pérdida de peso, con la consiguiente reducción de la sobrecarga mecánica, de los biomarcadores inflamatorios y del estrés oxidativo.

Palabras clave: Obesidad, Inflamación, Enfermedades Reumáticas.

\section{INTRODUÇÃO}

A obesidade tem sido descrita como uma condição inflamatória de baixo grau, associada ao aumento da produção de mediadores pró-inflamatórios como o fator de necrose tumoral alfa ou interleucina 6 e expressão alterada de adipocinas (NIKIPHOROU E e FRAGOULIS GE, 2018). O estilo de vida de grande parte da sociedade atual possui como pilares o sedentarismo e os maus hábitos alimentares. Essa combinação contribui para uma prevalência cada vez maior da obesidade, que já é considerada uma epidemia global (NIKIPHOROU E e FRAGOULIS GE, 2018). Apontada como uma doença metabólica crônica caracterizada pelo acúmulo de tecido adiposo, está presente tanto nos países desenvolvidos quanto nos em desenvolvimento (SILVA DCT, et al., 2013).

Essa condição possui propriedades inflamatórias e imunomoduladoras que afetam o organismo humano de diversas formas e, além de diminuir a mobilidade, também age através de mecanismos mais específicos, com consequências diretas no desenvolvimento e progressão de outras comorbidade. Assim, a obesidade se mostra como um possível fator agravante de doenças reumáticas e musculoesqueléticas, sendo elas: artrite reumatoide, artrite psoriática, espondilite anquelosante, lúpus eritematoso sistemico (NIKIPHOROU E e FRAGOULIS GE, 2018).

O acúmulo de gordura corporal a ponto de impactar negativamente a saúde costuma ser diagnosticado por meio de indicadores antropométricos como índice de massa corporal (IMC) e circunferência da cintura (CC). O peso corporal é rotineiramente registrado na prática clínica de reumatologistas, mas na maioria dos estudos sobre artrite reumatoide, esse parâmetro é usado apenas para caracterização de coorte demográfica (GUIMARÃES MFBR, et al., 2019).

Pessoas obesas normalmente possuem níveis elevados de marcadores inflamatórios circulantes, como proteína $C$ reativa (PCR), fator de necrose tumoral alfa (TNF- $\alpha$ ), interleucina 6 (IL-6) e inibidor 1 do ativador do plasminogênio (PAI 1). A associação documentada entre obesidade, aumento do risco cardiovascular $(R C V)$ e inflamação, bem como as alterações na composição corporal em pacientes com Artrite Reumatóide (AR), justificam investigar a influência da obesidade sobre essas doenças (GUIMARÃES MFBR, et al., 2019).

A osteoartrite $(\mathrm{OA})$ é a doença articular mais comum em todo o mundo, caracterizada pela perda progressiva da cartilagem articular, osteófitos e, frequentemente, sinovite. Os sintomas incluem dor e rigidez articular, resultando em dificuldade de mobilidade. Na patogênese dessa comorbidade, múltiplos tecidos articulares estão envolvidos: osso, sinóvia e cartilagem. A OA tem vários fatores de risco, incluindo predisposição genética, sexo e idade, mas o aumento da massa corporal é um importante fator preditor dessa doença (THIJSSEN E, et al., 2014). Ademais, destaca-se o acometimento do disco vertebral secundário a obesidade, o qual possui uma alta prevalência. Abrangendo tanto a sobrecarga mecânica, 
quanto as propriedades inflamatórias próprias desse perfil, as quais já foram previamente citadas (FERNÁNDEZ CR, et al., 2019).

Por conta da escassez de estudos realizados sobre o tema em questão, o objetivo desse artigo foi esclarecer a relação entre a obesidade, por meio do seu estado inflamatório crônico, na piora dos quadros de doenças reumatológicas através de uma revisão bibliográfica.

\section{REVISÃO BIBLIOGRÁFICA}

\section{Epidemiologia}

Em prospecção realizada, observou-se uma alta incidência de doenças articulares. Inclui-se nessa informação a osteoartrite, a qual acomete $80 \%$ da população acima de 60 anos e $70 \%$ acima de 70 anos (RIOFRIO RIV e SANCHEZ SGT, 2019). Ao analisar a prevalência de sobrepeso, foi observado que acomete $50,8 \%$ da população total brasileira, enquanto a obesidade atinge $17,5 \%$ (LINO JC, 2015).

No que tange a correlação do acometimento articular secundário à obesidade, em estudo realizado com 141 pacientes com IMC acima de 35 (obesidade mórbida), apontou-se para uma prevalência de sintomas de dor articular de 90,1\%. Já o diagnóstico de osteoartrite propriamente dito, por meio de avaliação radiológica realizada em 103 pacientes, foi observado que em 63,1\% dos casos houve acometimento do joelho, e em $40,8 \%$ do quadril. Além disso, destaca-se a maior perda funcional e a intensidade da dor maior em pacientes obesos (PACCA DM, et. al., 2018).

A partir da análise de outro estudo, com a seleção de 165 pacientes com diagnóstico de artrite reumatoide (AR), $40 \%$ apresentavam obesidade e $35,1 \%$ apresentavam sobrepeso. (PAATS A, et. al., 2018). Em estudo de caso-controle realizado na Suécia, dos 515 pacientes diagnosticado com AR, os indivíduos obesos apresentaram 3 vezes maior risco em comparação com indivíduos de peso normal (IANNONE F, et al., 2016).

Achados semelhantes puderam ser observados por outro estudo de caso-controle dinamarquês em 2748 pacientes com AR, ratificando essa forte relação com a obesidade e o alto IMC. E, por fim em estudo longitudinal com 109.896 mulheres matriculadas no Nurses Health Study-I (NHS-I) e 108.727 no Nurses Health Study - II (NHS-II), demonstrou que os riscos de AR soropositiva e soronegativa eram maiores entre mulheres com sobrepeso e obesas (IANNONE F, et al., 2016).

Em relação ao Lupus Eritematoso Sistêmico (LES), a prevalência aproximada é de 50 casos por 100.000 habitantes, acometendo, predominantemente, o sexo feminino, na relação de nove mulheres para um homem. A sintomatologia, comumente, inicia entre 20 e 40 anos de idade (LINO JC, 2015).

Finalmente, em estudo realizado com 170 pacientes diagnosticadas com LES atendidas no Ambulatório de Reumatologia do Hospital das Clínicas/UFMG, mostrou frequência da obesidade em 27,7\% das doentes e, 0 sobrepeso foi de $35,3 \%$. Em outro estudo realizado com 188 pacientes, evidenciou a frequência de excesso de peso $\left(I M C \geq 25,0 \mathrm{~kg} / \mathrm{m}^{2}\right)$ de $63,2 \%$ (119) e obesidade $\left(\mathrm{IMC} \geq 30,0 \mathrm{~kg} / \mathrm{m}^{2}\right.$ ) de $28,1 \%$ (53) em pacientes com LES (LINO JC, 2015).

\section{Fisiopatologia}

Evidências apontam que o tecido adiposo é um órgão endócrino e não somente um depósito de energia inerte. Esse órgão tem relação com o sistema nervoso central e é responsável por produzir hormônios e proteínas que estão relacionadas com processos imunes e inflamatórios (GUIMARÃES MFBR, et al., 2019).

Nesse sentido, a associação da obesidade com os eventos articulares é bem extensa. Destaca-se a ação de citocinas que são produzidas pelo tecido adiposo, sendo essas, adiponectina, resistina e leptina. Ademais, a maior carga que a articulação desse grupo é exposta, ocasiona resposta de mecanorreceptores localizados nos condrócitos, levando a ativação de citocinas, fatores de crescimento e metaloproteinases (PACCA DM, et al., 2018).

Em relação a essa exposição a carga anormal na obesidade, os condrócitos são levados a expressarem enzimas catabólicas, como as metaloproteinases de matriz (MMPs) - MMPs 1, 3 e 9 -, as quais estão 
associadas, principalmente a degradação da matriz cartilaginosa, inibindo a síntese de proteoglicanos e colágeno, sendo essas macromoléculas responsáveis pelo amortecimento articular. Além disso, os condrócitos submetidos a altas cargas têm expressão aumentada das citocinas pró-inflamatórias (IL) IL-1 $\beta$, TNF- $\alpha$ e da ciclooxigenase 2 (COX 2), o indutor de prostaglandina E2 (PGE2) (THIJSSEN E, et al., 2014).

Entretanto, acreditava-se que o desgaste articular estava associado exclusivamente ao aumento das cargas, levando ao desgaste mecânico das mesmas. Todavia, a combinação de inúmeros fatores, sejam eles mecânicos, metabólicos e/ou genéticos, desencadeiam o acometimento articular (SUN AR, et al., 2016; KULKARNI K, et al., 2016).

Salienta-se a importância do tecido adiposo para a secreção de fatores anti-inflamatórios, como a adiponectina e o secreted frizzled-related protein 5 (sFRP5), entretanto, esse controle está desregulado em pacientes obesos, o que leva a uma diminuição da secreção desses fatores e uma sobreposição de fatores pró-inflamatórios, como os citados previamente. Sabe-se que esse desbalanço de fatores anti-inflamatórios e pró inflamatórios é o fator determinante para a instalação da inflamação (LINO JC, 2015).

No que tange a ação das adiponectinas está relacionada com a ativação de fatores inflamatórios como a interleucina 6 e TNF- $\alpha$, que atuam diretamente na etiopatogênese da osteoartrite como mediadores próinflamatórios levando à destruição articular (QUENAN YE e OSORIO JH, 2017).

Como abordado sobre desregulação dos adipócitos vistos na obesidade, as células imunes têm um papel fundamental, principalmente os macrófagos. Tais células, componentes do sistema imune inato, apresentam um espectro de genótipos, tendo em seus extremos os macrófagos pró-inflamatórios (M1) e antiinflamatórios (M2). Os marcadores usados para detectar e classificar amplamente seus subtipos incluem aqueles de macrófagos inflamatórios (CD14, CD16, CD11b e toll - como receptores TLR 2 e 4) e macrófagos antiinflamatórios ou residentes (CD14, CD16, CD163, CD206 e CD11c) (RUDRAPATNA S, et al., 2019).

\section{Principais alterações relacionadas aos processos inflamatórios decorrentes da obesidade}

\section{Osteoartrite (OA)}

A osteoartrite é uma doença articular de evolução lenta, sendo a obesidade um dos fatores de risco (HERRERO-BEAUMONT G, et al., 2019). Pode-se dividir as articulações em articulações que sustentam peso (ASP), como a articulação do joelho, e articulações que não sustentam peso (ANSP), como as da mão. A sobrecarga demonstrou ter maior impacto na OA das articulações ASP, quando comparados com síndromes metabólicas. Era esperado que na OA em ANSP os principais fatores fossem bioquímicos/biológicos, como inflamação, em relação aos fatores biomecânicos, todavia não foi demonstrado tal relação (URBAN H e LITTLE CB, 2018).

A partir disso, destaca-se a osteoartrose de joelhos, a qual foi definida por Framinghan como decorrente da obesidade, junto aos fatores imutáveis como idade, sexo feminino e herança genética. Além da obesidade estar relacionada a gênese da patologia abordada, é pertinente salientar sua influência como fator de progressão. Estudos apontam que pacientes obesos possuem alterações radiográficas mais pronunciadas, o que é evidenciado pelo maior grau de dor que esse grupo apresenta. (LOURES, FB, et.al., 2015).

Além da obesidade, pacientes com OA apresentam outras comorbidades como Hipertensão Arterial Sistêmica, Diabetes Mellitus, Síndrome Metabólica, Depressão, entre outras. A identificação de tais comorbidades é importante, visto que o tratamento da OA pode causar uma piora no quadro dessas outras doenças subjacentes, decorrente da interação dessas comorbidades e OA e da terapia da osteoartrite (LEITE AA, et al., 2011).

\section{Artrite Reumátoide (AR)}

A AR é uma doença inflamatória crônica, sendo a principal manifestação do paciente a inflamação das articulações. Os linfócitos secretam citocinas recrutando células inflamatórias para o local acometido, podendo levar à deformação e, posterior, incapacidade. Ademais, é importante salientar que os adipócitos 
também secretam citocinas, contribuindo ainda mais para a progressão desse quadro (CASTILLOHERNANDEZ J, et al., 2016).

A obesidade, no que se refere à característica pró-inflamatória do tecido adiposo, parece estar vinculada à $A R$, visto que foi demonstrado uma maior atividade da $A R$ em pacientes obesos do que em pacientes não obesos (VIDAL C, et al., 2015). Além disso, no que tange a remissão da doença, pacientes obesos apresentam 40\% a menos de chance de remissão da patologia (LIU Y, et al., 2017).

\section{Artrite Psoriática (AP)}

Quanto a relação entre a artrite psoriática (AP) e a obesidade, há um aumento proporcional de sua incidência de acordo com o aumento do IMC. Além do que, a obesidade ainda interfere na eficiência do tratamento de AP, uma vez que não somente pode-se notar um aumento na suspensão do tratamento de pacientes em uso de inibidores de TNF, como também obesos, em comparação com não obesos, são menos propensos a atingir a atividade mínima da doença em 12 meses (NIKIPHOROU E e FRAGOULIS GE, 2018).

\section{Lúpus Eritematoso Sistêmico (LES)}

O Lúpus Eritematoso Sistêmico (LES) é uma doença inflamatória auto-imune, multissistêmica, resultado da conjugação de fatores genéticos, ambientais e hormonais que levam à perda do controle do equilíbrio e da imunorregulação celular. Dentro das comorbidades que frequentemente acompanham os pacientes com LES, destaca-se a obesidade, cuja prevalência vem crescendo na população geral e em pacientes com essa patologia (MARTINS SF, 2016).

Assim como o Lúpus Eritematoso Sistêmico, a obesidade também induz à inflamação crônica. Além disso, o acúmulo de gordura corporal leva a aumento dos níveis de citocinas pró-inflamatórias, que pode acarretar exacerbação da inflamação já presente no LES (LINO JC, 2015). Nesse sentido, a obesidade pode influenciar de forma negativa na resposta do tratamento da doença reumatológica (MARTINS SF, 2016).

\section{Gota}

Gota é uma artrite caracterizada pela deposição de cristais de monourato sódico na membrana sinovial, na cartilagem articular e nos tecidos periarticulares. Isto faz com que ocorra uma cascata inflamatória, resultando na secreção de várias citocinas pró-inflamatórias e o recrutamento de neutrófilos na articulação. Dentre os principais fatores de risco, inclui-se a obesidade, uma vez que ela contribui para o estado de hiperuricemia (BARROS SBC, et al., 2017).

\section{Degeneração do disco intervertebral}

O disco intervertebral é um componente que permite diversos movimentos da coluna vertebral, compreendendo uma região interna (núcleo pulposo), rico em proteoglicanos e colágeno tipo II, e uma região externa (ânulo fibroso) com matriz extracelular rica em colágeno tipo I e baixo conteúdo de proteoglicanos (FERNÁNDEZ CR, et al., 2019).

Em condições naturais, a homeostase entre síntese e degradação de matriz extracelular é mantida por citocinas e fatores de crescimento equilibrados. Durante cascatas degenerativas, a vascularização do núcleo pulposo e do ânulo fibroso permitem a migração de mastócitos e macrófagos para o disco e amplificação das cascatas inflamatórias, com consequente indução de dor lombar (FERNÁNDEZ CR, et al., 2019).

Mediadores inflamatórios como TNF- $\alpha$, IL-1 $\alpha / \beta$, IL-17, IL-6, Interferon- $\gamma$ e PGE-2 promovem a degradação da matriz extracelular, autofagia das células discais, senescência e apoptose. Os níveis de citocinas também se correlacionam com a sensação de dor nos pacientes, pelo aumento da Óxido Nítrico Sintase e consequentemente de óxido nítrico, o que sugere um feedback positivo na geração da dor (FERNÁNDEZ CR, et al., 2019). 
O estado pró-inflamatório induz também a expressão de fatores neurogênicos, como fator de crescimento neural (NGF) e fator neurotrófico derivado do cérebro (BDNF), que promovem o brotamento de fibras nervosas da raiz do gânglio dorsal até o núcleo pulposo e ânulo fibroso e aumento da sobrevida neural, como também da ação e sensibilidade dos canais de cátions associados a nocicepção nos gânglios de raízes dorsais. Tais mudanças levam a dissecção do disco, herniação e diminuição da altura do disco, prejudicando suas funções mecânicas e os tecidos adjacentes (FERNÁNDEZ CR, et al., 2019).

\section{Outras doenças Imunomediadas}

Na Espondilite Anquilosante, a adiposidade mostra uma correlação com resultados adversos da doença. Os quais envolvem, uma menor resposta aos tratamentos inibidores de TNF, Infliximabe ou adalimumabe. Pode se associar a obesidade a pior percepção dos benefícios do exercício. Contudo, não há evidências quando uma relação entre sua pior atividade e a obesidade (NIKIPHOROU E e FRAGOULIS GE, 2018).

Quanto a outras doenças imunomediadas, as informações ainda são bastante limitadas e faltam dados sobre as possíveis conexões entre a obesidade e outras doenças do tecido conjuntivo, incluindo a síndrome de Sjogren primária (NIKIPHOROU E e FRAGOULIS GE, 2018).

\section{O impacto da redução de peso na remissão das doenças}

Estudos apontam para o benefício da perda de peso visando a melhora sintomatológica, tendo efeito prolongado por até 1 ano após a redução. Esse processo deve-se à consequente redução de carga sobre a articulação, além de evidências apontarem uma redução de biomarcadores inflamatórios como IL-6 e PCR (URBAN H e LITTLE CB, 2018).

A maioria das diretrizes baseadas em evidências recomendam tratamento não cirúrgico e, se possível, tratamento não medicamentoso, dando prioridade à implementação de mudanças de estilo de vida. São recomendados exercícios em terra e aquáticos, estes com grande importância por reduzir a carga que incide diretamente sobre articulações acometidas por doenças reumatológica (ALRUSHUD AS, et al., 2017).

Por fim, demonstrou-se que a perda de peso por meio de dieta combinada ou não com exercício físico sobrepõem-se aos resultados quando comparados a apenas à prática de exercícios (URBAN H e LITTLE CB, 2018).

Não obstante, são necessários mais estudos com relevância adequada para que sejam avaliados os efeitos de uma intervenção combinada em comparação aos componentes aplicados de forma individual. A partir disso, observa-se a importância da redução de peso como tratamento não farmacológico para a melhora funcional e da dor articular (ALRUSHUD AS, et al., 2017).

\section{CONSIDERAÇÕES FINAIS}

A partir dos dados supracitados, conclui-se então, que a obesidade é um estado inflamatório do organismo e fator de risco para doenças auto imunes e reumáticas, como artrite reumatóide, artrite psoriática, lúpus eritemaotoso sistêmico, gota, degeneração do disco vertebral, espondilite anquilosante e outras. O tecido adiposo, antes considerado apenas como um armazém de energia inerte, vem se evidenciando como um órgão endócrino, capaz de produzir hormônios, proteínas, fatores inflamatórios e imunomoduladores, além do fator mecânico do peso, que, através de uma combinação multifatorial, acaba por comprometer as articulações, desencadeando e perpetuando essas doenças. O principal tratamento para a obesidade é a mudança no estilo de vida, tendo como primeira alternativa a adequação dietética, visando primeiramente a perda ponderal que retiraria os estímulos multifatoriais de modo que, fármacos e procedimentos cirúrgicos sejam utilizados apenas em casos específicos, repercutindo com diminuição das doenças reumatológicas e aumento na qualidade de vida desses pacientes. Porém, ainda são necessários maiores estudos sobre o benefício individual das intervenções combinadas, levando em conta a melhora funcional e a redução da dor articular. 


\section{REFERÊNCIAS}

1. ALRUSHUD AS, et al. Effect of physical activity and dietary restriction interventions on weight loss and the musculoskeletal function of overweight and obese older adults with knee osteoarthritis: a systematic review and mixed method data synthesis. BMJ Open, 2017; 7(6): e014537.

2. BARROS SBC, et al. Gota: aspectos básicos e principais complicações clínicas. Revista Multiprofissional em Saúde do Hospital São Marcos, 2018; 3(1): 32-40.

3. CASTILLO-HERNANDEZ J, et al. A obesidade é um determinante da resistência à insulina mais importante do que os níveis circulantes de citocinas pró-inflamatórias em pacientes com artrite reumatoide. Rev Bras Reumatol, 2017; 57(4): 320-329.

4. FERNÁNDEZ CR, et al. Molecular Relationships among Obesity, Inflammation and Intervertebral Disc Degeneration: Are Adipokines the Common Link? International Journal of Molecular Sciences, 2019; 20(8): 2030.

5. GUIMARAES MFBR, et al. High prevalence of obesity in rheumatoid arthritis patients: association with disease activity, hypertension, dyslipidemia and diabetes, a multi-center study. Adv. Rheumatol, 2019; 59(44).

6. HERRERO-BEAUMONT G, et al. Targeting chronic innate inflammatory pathways, the main road to prevention of osteoarthritis progression. Biochem Pharmacol., 2019; 165:24-32.

7. IANNONE F, et al. Impact of obesity in the clinical outcome of rheumatologic patients in biotherapy. Autoimmunity Reviews, 2016;15(5): 447-450.

8. KULKARNI K, et al. Obesity and Osteoarthritis. Maturistas, 2016; 89: 22-28.

9. LEITE AA, et al. Comorbidades em pacientes com osteoartrite: frequência e impacto na dor e função física. Revista Brasileira de Reumatologia, 2011; 51(2): 118-123.

10. LINO JC. Qualidade de vida relacionada à saúde em pacientes com lúpus eritematoso sistêmico atendidas no serviço de reumatologia do Hospital das Clínicas/UFMG: impacto da obesidade. Dissertação (Mestrado em Ciência de Alimentos) - Faculdade de Farmácia. Universidade Federal de Minas Gerais, Belo Horizonte, 2015; 129 p.

11. LOURES FB, et al. Evaluation of body mass index as a prognostic factor in osteoarthrosis of the knee. Rev. bras. ortop., 2016; 51(4): 400-404.

12. LIU Y, et al. Impact of Obesity on Remission and Disease Activity in Rheumatoid Arthritis: A Systematic Review and Meta-Analysis. Arthiritis Care Res (Hoboken). 2017; 69(2):157-165.

13. MARTINS SF, et al. Estado nutricional, bioquímico e consumo alimentar no Lúpus Eritematoso Sistêmico (LES): uma revisão de literatura. Monografia (Bacharel em Nutrição) - Departamento de Nutrição. Universidade Federal da Paraíba, João Pessoa, 2016; 35 p.

14. NIKIPHOROU E, FRAGOULIS GE. Inflammation, obesity and rheumatic disease: common mechanistic links. A narrative review. Ther Adv Musculoskelet Dis, 2018; 10(8): 157-167.

15. PAATS A, et. al, Impacto de la obesidad y su relación con la actividad de la enfermedad en pacientes con artritis reumatoide. Rev. parag. Reumatol., 2018; 4(2): 31-8.

16. PACCA DM, et al. Prevalence of joint pain and osteoarthritis in obese brazilian population. ABCD, Arq. bras. cir. dig., 2018; 31(1): e1344.

17. PORFIRIO E, FANARO GB. Collagen supplementation as a complementary therapy for the prevention and treatment of osteoporosis and osteoarthritis: a systematic review. Rev bras geriatr gerontol, 2016; 19(1):153-164.

18. QUENAN YE, OSORIO JH. Relación entre obesidad, adipocitoquinas y osteoatrosis: Una revisión. Univ. Salud, 2017; 19(3): 410-418.

19. RUDRAPATNA S, et al. Obesity and muscle-macrophage crosstalk in humans and mice: A systematic review. Obesity review, 2019; 20(11): 1572-1596.

20. SILVA DCT, et al. Influencia do tempo de exposicao a obesidade induzida por dieta hiperlipidica sobre os colagenos tipo I e III miocardico. Arq. Bras. Cardiol., 2014; 102(2): 157-164.

21. SUN AR, et al. Is Synovial Macrophage Activation the Inflammatory Link Between Obesity and Osteoarthritis? Curr Rheumatol, 2016; 18(9): 57.

22. THIJSSEN E, et al. Obesity and osteoarthritis, more than just wear and tear: pivotal roles for inflamed adipose tissue and dyslipidemia in obesity-induced osteoarthritis. Rheumatology (Oxford), 2015; 54(4): 588-600.

23. URBAN H, LITTLE CB. The role of fat and inflammation in the pathogenesis and management of osteoarthritis. Rheumatology (Oxford), 2018; 57(4): 10-21.

24. RIOFRIO RIV, SANCHEZ SGT. Caracterización clínico epidemiológica de la osteoartritis en el Cantón Colt. Rev Cuba Reumatol, 2019; 21(2): e82.

25. VIDAL C, et al. Association of Body Mass Index Categories with Disease Activity and Radiographic Joint Damage in Rheumatoid Arthritis: A Systematic Review and Metaanalysis. J Rheumatol, 2015; 42(12): 2261-2269. 